Biometrics 28, 499-518

June 1972

\title{
THE ALLOCATION OF RESOURCES IN POPULATION SCREENING: A DECISION THEORY MODEL
}

\author{
H. Goldstein \\ National Childrens Bureau, 1 Fitzroy Square, London, W1P 5AH, England
}

\section{SUMMARY}

In the medical screening of a population for latent diseases, it is often possible to define subpopulations with differing 'risks' of diseases. In order to make the most efficient use of the resources available for screening programmes, the allocation of these resources should take account of the differing risks. Some decision theory models are proposed for determining the optimum allocations.

These models are applied to data on handicaps in young children and on perinatal mortality.

\section{INTRODUCTION}

Recent years have seen the development of many inexpensive, easily administered techniques for detecting diseases. The availability of these has often stimulated mass screening programmes, some of the more familiar ones being those for cervical and lung cancer.

These programmes have operated in a variety of ways. In some cases the screening facilities have simply been advertised and the public invited to attend. In other cases, for example in schoolchildren, all individuals of a particular age group have been examined, and enlightened employers have sometimes screened their total work force. There has also been a recognition, however, that in cases where the screening resources are relatively scarce, for example in specialised ante-natal care (Aubrey and Nesbitt [1969]), the available resources should be concentrated on those individuals known or thought to be at higher-than-average risk of developing the disease screened for.

In the early 1960's this thinking led to the setting up of 'At Risk' registers of children in certain English local authorities (Lindon [1961]). The children on these registers were those identified at 'high risk' of physical or mental handicap on the basis of factors ascertained at birth. The intention was that these children would then be given special attention during infancy and in particular be given priority in any screening programmes. The remainder of this paper will discuss the allocation of resources in the context of the detection of handicaps in children, although the mathematical results are applicable to other situations.

In a recent paper, Alberman and Goldstein [1970] discussed the 'At Risk' 
registers and presented a simple decision theory model to determine resource allocation. They only considered, however, the case of a single type of handicap and a single type of resource used to detect it. The present paper extends this model to the case of more than one type of handicap and resource for detection. In the next section, some general considerations are discussed and this is followed by the development of models for particular situations with some numerical examples.

\section{GENERAL CONSIDERATIONS}

It will be supposed in what follows that in a population of children at a given age it is possible to define different handicaps. In addition, it is supposed that the probabilities of finding these handicaps in a randomly chosen child are given by functions of 'predictor' variables measured at an earlier age. The handicaps of most interest are those which are severe enough to make it necessary for the child to need special education or care and where early detection is useful. The predictor variables are those social, maternal, and obstetric ones which can be measured at the birth of the child. A detailed discussion of the problems of selecting predictor variables and of defining handicaps is given by Alberman and Goldstein [1970]. In that paper a linear model was used relating the logit transformation of the probability of handicap to 5 predictor variables, each present at either 2 or 3 levels.

This model may be extended to the multivariate case of more than one handicap, by defining a 'multivariate logit' as follows:

$$
\begin{aligned}
P_{i i} & =e^{Z_{i i}} / D_{i}, \\
D_{i} & =\sum_{i} e^{Z_{i i}}, \quad i=1, \cdots, s \quad j=1, \cdots, k
\end{aligned}
$$

where $P_{j i}$ is the probability of the $j$ th handicap in the $i$ th group and $Z_{j i}$ is a linear function of independent variables. The model may be written

$$
\mathbf{Z}=\mathbf{X} \cdot \mathbf{B} \cdot \mathbf{A} \text {, }
$$

where $\mathrm{B}$ is a $q \times t$ matrix of unknown coefficients, $\mathbf{X}$ is a $n \times q$ 'design matrix' (rank $q$ ) defining the independent variables, and $\mathbf{A}$ is a $t \times k$ 'design matrix' (rank $t$ ) for the ways of classification among the probabilities of handicap.

The maximum likelihood (ML) analysis of this model is described in detail by Bock [1969]. Alternative models have been proposed (Goodman [1970]) but have not been tried on these data.

Following an analysis using equation (1) the population can be divided into $s$ groups each one defined by a combination of levels of the predictor variables. For an individual (child) in the $i$ th group let the estimated probability of having the $j$ th type of handicap be $p_{i i}$. Also let the proportion of individuals in the $i$ th group be $a_{i}$.

$$
\sum_{i} a_{i}=1
$$

The case $s=2$ with just a 'high risk' and 'low risk' group is of special interest and will be returned to later. 
Suppose that there are certain resources available for screening the population in order to try to detect the handicaps. Resources for screening may take the form of tests for defective vision, hearing, motor functioning and so on. It is assumed that for each type of handicap there is a corresponding type of resource for detecting it. Furthermore, it will be assumed that the resources can be divided and allocated to the individuals in the population in any manner.

Let the amount of resource $j$ given to each individual in group $i$ be $R_{i i}$. If there are $T_{i}$ individuals in group $i$, then the total amount of resource $j$ which is allocated is $T Q_{i}$ :

$$
\sum_{i=1}^{8} T_{i} R_{i i}=T Q_{i}
$$

where $\sum_{i=1}^{i} T_{i}=T$ so that $a_{i}=T_{i} / T$.

If there is a given total amount of resource $j$ then the value of $Q_{i}$ is fixed. Alternatively, the total amount $T Q$ of all resources may be fixed in which case

$$
\begin{gathered}
\sum_{i} \sum_{i} w_{i} a_{i} R_{i i}=Q \\
\sum_{i} w_{i}=1
\end{gathered}
$$

The $w_{i}$ are introduced to convert the different units in which the separate resources may be measured to a common unit, for example a monetary one (see below).

The choice of a utility function $F(x)$ to evaluate the screening procedure, will in part depend on the situation in which the model is being applied and in particular on the facilities available to deal with the handicaps which are detected.

The simplest natural choice for such a function is as follows:

$$
F(x)=\begin{array}{ll}
1 \text { if a handicap is detected } \\
0 \text { otherwise }
\end{array}
$$

The different handicaps may, however, be given different weights. For example, it may be felt more important to detect a speech defect than a visual defect at a given age, in which case (5) may be modified to

$$
F(x)=\begin{array}{ll}
u_{i} & \text { if handicap } j \text { detected } \\
0 & \text { otherwise }
\end{array}
$$

Alternatively, instead of being defined solely in terms of the presence or absence of a handicap, the utility function may take account of the association between the handicaps leading to a function of the form:

$$
F(x)=\begin{array}{ll}
1 & \text { if a child has one or more handicaps } \\
0 & \text { otherwise }
\end{array}
$$


which is concerned solely with the number of children handicapped in any way. More generally $F(x)$ will be some function of the numbers of the different types of handicaps per child. An intermediate function between (5) and $(7)$ is $F(x)=u \log (1+x)$, where $x$ is the number of handicaps detected in a child, and $u$ is an arbitrary constant.

In the remainder of this paper only functions (5) and (6) will be considered, since these lead to relatively simple equations and seem to be reallistic for many practical situations.

Using (6) the expected utility for the $j$ th handicap in an individual in the $i$ th group is

$$
T a_{i} u_{j} p_{j i} g\left(R_{j i}\right),
$$

where $g\left(R_{j i}\right)$ is the probability of detecting the $j$ th handicap in an individual in the $i$ th group when that individual possesses, or will develop the handi$\operatorname{cap} j$.

Thus the total expected utility is

$$
C=T \sum_{i} \sum_{i} a_{i} u_{i} p_{i i} g\left(R_{j_{i}}\right) .
$$

The $R_{j i}$ are determined by maximising $C$ for variation in the $R_{i i}$. In order to do this, however, a form for $g$ must be chosen. The following assumptions about this form will be made:

(a) $g\left(R_{j i}\right)$ does not depend on $i$, so that the probability of detecting a handicap if one is present or 'latent' is unrelated to the risk of possessing that handicap.

(b) $g\left(R_{j i}\right)$ and $g\left(R_{k i}\right)$ are independent $(j \neq k)$ so that the detection of one handicap does not influence the detection of others.

Assumption (a) may not always be very realistic, since the chance of having a handicap may well be related to the aetiology of the handicap, in which case the detection of the handicap may be easier in one child than in another. There does not seem to be any empirical information which casts light on this problem. A special case where assumption (a) is not made is given in Model 5 .

As for assumption (b), it may be true that a given handicap is easier or more difficult to detect if others have previously been detected, but again there seems to be no useful information available on this.

An explicit form of $g\left(R_{i i}\right)$ has still to be determined, and one possible choice can be motivated as follows:

Suppose the resources devoted to an individual $\left(R_{j i}\right)$ are used in discrete amounts (e.g., a unit amount is one screening test), and the probability of one unit amount failing to detect a handicap is $q$. Then for $x$ units the probability, assuming independence, of $x$ units of resource failing to detect a handicap is $q^{x}=q^{b R_{i i}}$, where $b$ is a constant. Therefore the probability of detection is $1-q^{b R_{i i}}$. Thus if the unit of $R_{i i}$ is suitably chosen, the probability of detection is

$$
1-e^{-R_{i i}}
$$


The unit of $R_{j i}$ is thus determined by the probability of detection, so that different resources will not all necessarily be measured in the same units. Since the resources are all positive, there is the inequality constraint

$$
R_{i i} \geq 0 \text { all } i, j \text {. }
$$

Specific models will now be considered in detail. In Models 1 to 4 the above form of $g\left(R_{i i}\right)$ will be assumed and in Model 5 a different form applicable to certain situations will be examined.

\section{MODEL 1}

In the previous section, alternative utility functions and alternative constraints on the amounts of resources were discussed. The first model to be considered uses the constraint given by equations (3) and (6), namely a fixed amount of resource for each handicap and the utility function given by (6).

Since the $u_{i}$ are relative utilities we may without loss of generality define

$$
\sum_{i} u_{i}=k \quad u_{i}>0 .
$$

The total expected utility is thus

$$
C=T \sum_{j} \sum_{i} a_{i} u_{j} p_{i i}\left(1-e^{-R_{j i}}\right) .
$$

This expression is to be maximized with respect to the $R_{j i}$ subject to (3) which can be written

$$
\sum_{i} R_{j_{i}} a_{i}=Q_{i} \quad j=1, \cdots, k
$$

and also subject to (10). Ignoring (10) the unconditional maximum or minimum of $C$ is given by

$$
\partial C / \partial R_{j i}+\lambda_{j} a_{i}=0,
$$

where $\lambda_{j}$ is a Lagrange multiplier giving

$$
u_{j} p_{j i} e^{-R_{j i}}+\lambda_{j}=0
$$

or

$$
R_{j i}=\log p_{i i}+B_{j}^{\prime}+\log u_{i} .
$$

Multiplying by $a_{i}$ and summing over $i$ for each value of $j$ gives

$$
B_{i}^{\prime}=Q_{i}-\sum_{i} a_{i} \log p_{j i}-\log u_{i},
$$

so that

$$
R_{j i}=\log p_{j i}+Q_{i}-\sum_{i} a_{i} \log p_{i i}
$$

and

$$
C / T=\sum_{i} u_{i} p_{i 0}-\sum_{i} u_{j} e^{-Q_{i}} \prod_{i} p_{j_{i}}^{a_{i}}
$$


where

$$
p_{i 0}=\sum_{i} a_{i} p_{i i},
$$

which is the proportion of individuals with the $j$ th handicap in the population. $C / T$ is the average (maximised) number of handicaps detected per individual.

The above solution is readily shown to be a maximum, by noting that all the second order differentials are negative:

$$
\partial^{2} C / \partial R_{i i}^{2}=-a_{i} u_{j} p_{j i} e^{-R_{i i}}<0 .
$$

when $a=1$ and $s=1$, then

$$
\begin{aligned}
C / T & =\sum_{i} u_{i} p_{j 0}-\sum_{i} u_{j} p_{i 0} e^{-Q_{i}} \\
& =\sum_{j} u_{i} p_{j 0}\left(1-e^{-Q_{i}}\right)=\sum_{i} C_{i} \text { (say). }
\end{aligned}
$$

For the $j$ th handicap,

$$
C_{j}^{\prime}=C_{i} / p_{j 0}=u_{j}\left(1-e^{-Q_{j}}\right)
$$

is the proportion of all children with handicap $j$ in which the handicap is detected, when each child in the population receives the same amount of resource $j$. If this is known in a particular population, the $Q_{i}$ may be determined using (21) and substituted in equations (16)-(18). The numerical results given later are evaluated for ranges of values of the $C_{i}^{\prime}$.

If $k=1$, equations (19) and (20) reduce to the formulae given by Alberman and Goldstein [1970] for a single handicap. The solutions for Model 1 may be regarded as the sum of the separate solutions for single handicaps, the only restriction in this case being that the combinations of predictor variables are the same for each handicap.

For small values of $P_{j i}$ and $Q_{i}$, equation (18) may give some negative values of $R_{i i}$. When this occurs it becomes necessary to search for a relative maximum subject to (10). It is shown in the Appendix that this relative maximum occurs when one or more of the $R_{i i}$ are equal to zero, for each resource containing a negative $R_{j i}$.

If $r$ is the number of resources with at least one $R_{i i}$ negative, then if $s$ and $r$ are not too large it is a straightforward matter to evaluate $C / T$ for all of the $2 r\left(2^{s-1}-1\right)$ possibilities and to choose the maximum. Equations (15)-(17) are modified by omitting the $R_{i i}$ which are set equal to zero.

Where complete evaluation is not feasible, solutions can be sought using non-linear programming techniques (see, e.g., Geary and McCarthy [1964]).

In this and the following models it is worth pointing out that some of the $p_{j i}$ may be equal and so also may some of the $R_{i i}$, thus allowing consideration of special cases where different resources may be detecting the same handicap or one resource may be detecting different handicaps. 


\section{MODEL 2}

In practice, it is likely that further restrictions may have to be placed on Model 1. For example, when a standard battery of screening tests for the different handicaps is available, then the relative amount of each test is fixed and this restriction gives the $k-1$ constraints

$$
R_{i i}-d_{i} R_{i+1, i}=0 \quad d_{i}>0
$$

or

$$
\sum_{i} a_{i} R_{i i}-d_{i} \sum_{i} a_{i} R_{i+1, i}=0,
$$

and using (3) this gives

$$
d_{i}=Q_{i} / Q_{i+1} .
$$

All $R_{j i}(j=2, \cdots, k)$ can now be expressed in terms of $R_{1 i}, Q_{1}, \cdots, Q_{k}$. Maximising $C$ as before, we have

$$
\partial C / \partial R_{1 i}+\lambda a_{i}=0 \quad i=1, \cdots, s,
$$

giving

$$
\sum_{i} u_{i} p_{i i} \exp \left(-R_{1 i} Q_{i} / Q_{1}\right)+\lambda=0
$$

which together with $\sum_{i} a_{i} R_{1 i}=Q_{1}$ can be solved iteratively. For $s=2$, (26) becomes

$$
\begin{aligned}
\sum_{i} u_{i} p_{i 1} Q_{i} \exp (- & \left.R_{11} Q_{i} / Q_{1}\right) \\
& -\sum_{i} u_{i} p_{i 2} Q_{i} \exp \left[-Q_{i}\left(Q_{1}-a_{1} R_{11}\right) /\left(a_{2} Q_{1}\right)\right]=0 .
\end{aligned}
$$

As a starting value for the solution of (27), $R_{11}=Q_{1}$ can be used. When $s=1$, then $R_{i i}=Q_{j}$ and

$$
C / T=\sum_{i} u_{i} p_{i 0}\left(1-e^{-0_{i}}\right) .
$$

The $Q_{i}$ may now be determined as described in model 1 . The constraint (10) becomes $R_{1 i} \geq 0$ since $d_{i}>0$.

The relative maximum where one or more $R_{1 i}$ are less than zero occurs when one or more $R_{1 i}$ are equal to zero (see Appendix) and a similar procedure to that described in Model 1 may be used to obtain the maximum.

\section{MODEL 3}

It may often be the case that a given total amount of resource is available for detecting all handicaps, rather than the amount for each handicap being fixed in advance. This is the situation described by equation (4).

The total amount of resource will be measured in some common unit whereas the resources specific to each handicap will in general be measured 
in different units which are determined by the proportion of each type of handicap that a given amount of the total resource will detect. The $w_{i}$ defined in (4) are therefore introduced as linear conversion factors, and are calculated as follows.

If all the available resources $T Q$ are allocated to detecting handicap $j$ with $s=1$, then using (21) the number of handicaps detected is

$$
C_{i}=T p_{j 0}\left(1-e^{-Q / w_{i}}\right)
$$

The quantity $C_{i} /\left(T p_{i 0}\right)=D_{i}$, say, is the proportion detected of the total number of $j$ th handicaps, using all resources. If the $D_{j}$ are known in a particular population then (4) and (28) determine $Q$ and the $w_{i}$ :

$$
\begin{aligned}
Q & =-\left\{\sum_{i}\left[\log \left(1-D_{i}\right)\right]^{-1}\right\}^{-1}, \\
w_{i} & =-Q\left[\log \left(1-D_{i}\right)\right]^{-1} .
\end{aligned}
$$

The total expected utility is

$$
C=T \sum_{j} \sum_{i} u_{j} a_{i} p_{i i}\left(1-e^{-R_{j i}}\right),
$$

where the $u_{i}$ are assumed known, as before. Maximising $C$ gives

$$
\begin{gathered}
\partial C / \partial R_{j i}+\lambda w_{j} a_{i}=0 \\
R_{j i}=\log p_{j i}-\log w_{j}+B^{\prime}+\log u_{j} .
\end{gathered}
$$

Multiplying by $a_{i} w_{j}$ and summing over $i, j$ gives

$$
B^{\prime}=Q-\sum_{i} a_{i} \sum_{i} w_{i} \log p_{j i}+\sum_{i} w_{i} \log w_{i}-\sum_{i} w_{i} \log u_{i}
$$

which leads to

$C / T=\sum_{i} \sum_{i} a_{i} u_{i} p_{i i}-\exp \left(-Q+\sum_{i} \sum_{j} a_{i} w_{i} \log p_{i i}\right) \prod_{j}\left(w_{j} / u_{j}\right)^{-w_{j}}$.

For $s=1$

$$
C / T=\sum_{i} u_{i} p_{i_{\diamond}}-e^{-Q} \prod_{j}\left[w_{i} /\left(p_{j 0} u_{i}\right)\right]^{-w_{i}}
$$

\section{MODEL 4}

This model is effectively a combination of Models 2 and 3, being obtained by adding the constraint (23) to Model 3.

The $w_{i}$ and $Q$ are determined by equations (29) and (30) and the $u_{i}$ are assumed known as before. As in Model 2 the $R_{i i}$ can be expressed in terms of $R_{1 i}$ and $d_{1}, \cdots, d_{k-1}$ which must now be determined.

Maximising $C$ gives

$$
\partial C / \partial R_{y_{i}}+\lambda a_{i} \sum_{m} w_{m} D_{m}=0 \quad i=1, \cdots, s
$$


where

$$
D_{m}=\left(\prod_{j=0}^{m-1} d_{j}\right)^{-1} \quad d_{0}=1
$$

and

$$
\partial C / \partial d_{j}+\lambda R_{1 i} a_{i} \sum_{m=j+1}^{k} w_{m} D_{m} / d_{j}=0 \quad j=1, \cdots, k-1,
$$

which leads to

$$
\sum_{m=1}^{k} D_{m} u_{m} p_{m i} e^{-R_{1 i} D_{m}}+\lambda \sum_{m=1}^{k} w_{m} D_{m}=0
$$

and

$$
\sum_{m=j+1}^{k} u_{m} p_{m i} e^{-R_{1 i} D_{m}}+\lambda \sum_{m=j+1}^{k} w_{m} D_{m}=0,
$$

which together with (4) and (31) can be solved iteratively.

When $k=1$ these equations give

$$
p_{1 i} e^{-R_{1 i}}+\lambda=0 \quad i=1, \cdots, s
$$

which is equivalent to Model 1 with $k=1$.

\section{MODEL 5}

The form of $g(R)$ given by equation (9) assumes, among other things, that the resources can be measured in continuous units and that any amount may be given to an individual. In many situations this may be a reasonable approximation, but there are some circumstances where this clearly does not hold. In these cases the resource can only be allocated in one of a finite number of fixed amounts. In the extreme case, for example, it may only be possible to allocate either a given fixed amount or none at all. Such a resource will be referred to as dichotomous and in the general case we shall consider polytomous resources.

An example of such a polytomous resource is the type of provision given to a woman for childbirth. A baby will generally be delivered in a hospital maternity unit, in a general practitioner unit, or at home. If for example, the aim is to prevent the death of the baby within the first week of life, then there will be different probabilities of preventing such a 'perinatal' mortality associated with the three places of delivery. The problem is then that of allocating mothers to one of these places based on a known risk of perinatal mortality.

The general case of more than one type of resource for more than one handicap can be reduced to the case of a single type of 'pseudo-resource' for more than one handicap by considering all possible combinations of the different amounts or levels of each resource. This device covers in principle the situations analogous to equations (3) and (4) where either the total amount of each resource is fixed or the total amount of all resources is fixed. 
The situation where some resources are polytomous and some are continuous will not be dealt with in detail, save to remark first that in the case of Model 1 the resources are effectively treated separately so that no new problems arise. Second, when the total amount of all resources is fixed a solution can be obtained, in principle, by considering all possible ways of allocating levels of the pseudo-resource and then for each of these solving for the continuous resources; and selecting the maximum among these solutions. Other methods of tackling the problem might consist either of imposing a continuous scale on the pseudo-resource or polytomising the continuous resources.

Suppose then that there is a pseudo-resource which can be allocated at one of $m$ fixed levels. Let the probability of detecting the $j$ th handicap with level $r$ in the $i$ th group be

$$
\begin{aligned}
r & =1, \cdots, m \\
g_{i i}(r)=g_{r i} \quad j & =1, \cdots, k . \\
i & =1, \cdots, s
\end{aligned}
$$

We now have to consider the two cases analogous to equations (3) and (4). First we may fix the total number of units of each level so that

$$
\sum_{i \varepsilon R_{r}} a_{i}=Q_{r}
$$

summation taking place over those groups where level $r$ has been allocated (the set $R_{r}$ ).

Second, we may fix the grand total number of units and (39) becomes

$$
\sum_{r} \sum_{i \varepsilon R_{r}} a_{i} w_{r}=Q
$$

where the $w_{r}$ are linear conversion factors as before. The solution using (40) will not be considered in detail, but can in principle be reduced to (39) by considering all possible combinations of different $Q_{r}$, obtaining the solution for each combination as for (39), and selecting the maximum among these.

If every individual in the $i$ th group receives level $r$ then the expected number of handicaps detected in this group is

$$
\sum_{i} T_{i} p_{i i} g_{r i i}
$$

with $T_{i}$ and $P_{i i}$ as before. The quantity to be maximised is therefore

$$
C=T \sum_{i} \sum_{i} a_{i} p_{i i} g_{r i i}
$$

subject to (39). We shall further assume, without loss of generality, that the total number of units of resource is equal to the total number of individuals so that

$$
\sum_{r} Q_{r}=1
$$

Some of the $Q_{r}$ may be zero.

Consider any allocation of levels to individuals, and assume that all the 
individuals in one group receive the same level of resource. (This may be achieved by arbitrarily dividing up any group in which this is not so into several groups in which it is). Any reallocation of resources will involve the exchange of levels between individuals in different groups.

Suppose that a level $r$ from an individual in the $i$ th group is exchanged with a level $r^{\prime}$ from an individual in the $i^{\prime}$ th group. The loss in the expected number of handicaps detected is

$$
\begin{aligned}
G & =\sum_{i}\left[\left(p_{i i} g_{r^{\prime i}}+p_{i^{\prime}} g_{r^{\prime} i^{\prime}}\right)-\left(p_{i i} g_{r^{\prime} j i}+p_{i^{\prime}} g_{r^{\prime} i^{\prime}}\right)\right] \\
& =\sum_{i}\left[p_{i i}\left(g_{r^{i} i}-g_{r^{\prime} j i}\right)-p_{j i}\left(g_{r^{\prime} i^{\prime}}-g_{r^{\prime} i^{\prime}}\right)\right] .
\end{aligned}
$$

In order for the new allocation to result in a greater number of handicaps detected we require $G<0$ or

$$
\sum_{i} p_{i i}\left(g_{r^{i} i}-g_{r^{\prime} j i}\right)<\sum_{i} p_{i i}\left(g_{r g i^{\prime}}-g_{r^{\prime} i^{\prime}}\right) .
$$

Thus, starting with any given allocation we may proceed by considering all possible exchanges, choosing that exchange which gives the minimum value of $G$, and repeating the process. Given a suitable allocation to start with, this procedure should converge to the solution fairly rapidly. One way of obtaining a suitable starting allocation would be to consider first of all the highest risk group (i.e. the group with the largest value of $\sum_{i} p_{i i}$ ) and allocate to individuals in this group level $r$ such that $p_{j i} g_{r i i}$ is maximised. This is then repeated for the next highest risk group and so on until all the resources have been allocated. This initial allocation will in many situations also be the optimum allocation.

This procedure may be illustrated simply, by considering the case of one type of handicap $(k=1)$. Equation (44) now becomes, dropping the suffix $j$,

$$
\begin{gathered}
p_{i}\left(g_{r i}-g_{r^{\prime} i}\right)<p_{i^{\prime}}\left(g_{r i^{\prime}}-g_{r^{\prime} i^{\prime}}\right), \\
p_{i} / p_{i^{\prime}}<\left(g_{r^{\prime}}-g_{r^{\prime} i^{\prime}}\right) /\left(g_{r i}-g_{r^{\prime} i}\right) .
\end{gathered}
$$

The condition for the above initial allocation to be the optimum allocation is that if $p_{i} \geq p_{i^{\prime}}$, then

$$
p_{i} / p_{i^{\prime}} \geq\left(g_{r i^{\prime}},-g_{r^{\prime} i^{\prime}}\right) /\left(g_{r i}-g_{r^{\prime} i}\right) .
$$

Equation (46) may be satisfied in a number of ways. One of these is the special case

$$
g_{r i}=g_{r i}, \text { for all } r
$$

and we require

$$
p_{i} / p_{i^{\prime}} \geq\left(g_{r i}-g_{r^{\prime} i^{\prime}}\right) /\left(g_{r i}-g_{r^{\prime} i^{\prime}}\right)=1
$$

which is true since $p_{i} \geq p_{i}$.

More generally (46) is satisfied if

$$
g_{r i}-g_{r^{\prime} i}=D p_{i}^{x}, \quad x>-1, \quad D>0,
$$


In practice it will often be reasonable to assume that

$$
g_{r i}{ }^{\prime}-g_{r^{\prime} i} \geq g_{r i}-g_{r^{\prime} i}
$$

which also satisfies (46).

It should be noted that we are no longer assuming that the probability of detecting a handicap with a given amount of resource is the same for each risk group. This assumption is, in fact, the special case given by (47).

\section{ILLUSTRATIVE EXAMPLES}

In order to see what the effect of different restrictions are, Models 1-3 will be compared using data from the National Child Development Study. The exact definitions of the variables used in this example and why they were chosen are given in a recent publication of the study by Davie et al. [1972]. An example of the use of Model 5 is given using data from the Perinatal Mortality Survey (Butler and Alberman [1969]). Two types of handicaps are considered, 'severe physical or mental handicap' with a prevalence among 7 -year olds of 11.6 per 1000 and 'educational handicap' with a prevalence of 22.3 per 1000 .

The case $s=2$, which is of much practical interest, will be discussed in detail. In this case children are classified as either 'high' or 'low' risk on the basis of the 'perinatal' variables measured at birth listed in Table A.

\section{TABLE A}
1. Neonatal illness
2. Parity
3. Social class

4. Method of delivery

5. Birthweight-gestation (completed weeks)
(a) Ill during first week of life
(b) Not ill during first week of life
(a) Parities 4 and over
(b) Parities 0-3
(a) Social class 5

$$
\begin{aligned}
& \begin{array}{l}
\text { or no male } \\
\text { head of house- } \\
\text { hold } \\
\text { Social class 1-4 }
\end{array} \\
& \text { General } \\
& (1951)
\end{aligned}
$$
(b) Social class 1-4

(a) Breech, face, shoulder, internal
version, or unattended delivery. (Abnormal)

(b) Remainder. (Normal)

(a) Less than 2,500 g. or less than 37 weeks (Premature)

(b) Over 2,500 g. and over 42 weeks. (Postmature)

(c) Over 2,500 g. and 37-42 weeks (Normal)

These perinatal variables are then related to the probabilities of the two types of handicap at 7 years, using the multivariate logit model described earlier.

The design matrix for the independent (perinatal) variables is of rank 7 , 
that is, a 'main effects' design, and the design matrix for the ways of classification among the probabilities of handicap is of rank 2, also a 'main effects' design. The results are given in Table 1 , which presents tests of significance for the effects of each of the perinatal variables on the joint probabilities of handicap.

All the perinatal variables have significant effects, although as can be seen from the values of some of the individual standard errors, this is sometimes due entirely to one or other of the handicaps. This fact that the different types of handicap are 'predicted' by different sets of perinatal variables is discussed further in Davie et al. [1972] where the two types of handicap are treated completely separately. For the purposes of the example, however,

\section{TABLE 1}

Fitted Constants and Analysis of Variance

(All chi square values are adjusted for the other factors)

For definitions of perinatal variables see Table A

\begin{tabular}{|c|c|c|c|c|c|c|}
\hline \multicolumn{3}{|c|}{ Source } & $\begin{array}{l}\text { Fitted } \\
\text { Constant }\end{array}$ & $\begin{array}{l}\text { Standard } \\
\text { Error }\end{array}$ & D.F. & $x^{2}$ \\
\hline Overall & $\left\{\begin{array}{l}\text { Severe Handicap } \\
\text { Educational Handicap }\end{array}\right.$ & & $\begin{array}{l}-1.552 \\
-1.114\end{array}$ & & & \\
\hline $\begin{array}{l}\text { Neonatal } \\
\text { Illness }\end{array}$ & $\left\{\begin{array}{l}\text { Severe Handicap } \\
\text { Educational Handicap }\end{array}\right.$ & $\begin{array}{l}\text { a) }- \text { b) } \\
\text { a) }- \text { b) }\end{array}$ & $\begin{array}{l}0.633 \\
0.268\end{array}$ & $\begin{array}{l}0.180 \\
0.178\end{array}$ & 2 & $11.1^{* *}$ \\
\hline Parity & $\left\{\begin{array}{l}\text { Severe Handicap } \\
\text { Educational Handicap }\end{array}\right.$ & $\begin{array}{l}\text { a) }-b) \\
\text { a) }-b)\end{array}$ & $\begin{array}{l}0.280 \\
0.652\end{array}$ & $\begin{array}{l}0.126 \\
0.074\end{array}$ & 2 & $67.0 * * *$ \\
\hline Social Class & $\left\{\begin{array}{l}\text { Severe Handicap } \\
\text { Educational Handicap }\end{array}\right.$ & $\begin{array}{l}\text { a) }-b) \\
\text { a) }-b)\end{array}$ & $\begin{array}{l}0.015 \\
0.566\end{array}$ & $\begin{array}{l}0.135 \\
0.071\end{array}$ & 2 & $53.5^{* * *}$ \\
\hline Delivery & $\left\{\begin{array}{l}\text { Severe Handicap } \\
\text { Educational Handicap }\end{array}\right.$ & $\begin{array}{l}\text { a) }- \text { b) } \\
\text { a) }- \text { b) }\end{array}$ & $\begin{array}{l}0.398 \\
0.211\end{array}$ & $\begin{array}{l}0.150 \\
0.122\end{array}$ & 2 & $8.5^{*}$ \\
\hline $\begin{array}{l}\text { Birthweight } \\
\text { Gestation }\end{array}$ & $\left\{\begin{array}{l}\text { Severe Handicap } \\
\text { Educational Handicap }\end{array}\right.$ & $\begin{array}{l}\text { a) } \\
\text { b) } \\
\text { c) } \\
\text { a) } \\
\text { c) }\end{array}$ & $\begin{array}{r}0.121 \\
-0.013 \\
-0.108 \\
0.155 \\
0.030 \\
-0.185\end{array}$ & & 4 & $11.9^{*}$ \\
\hline
\end{tabular}

Test for 'goodness of fit' of model: $\chi^{2}=126.7$ D.F. $=97^{*}$

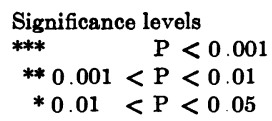




\begin{tabular}{|c|c|}
\hline 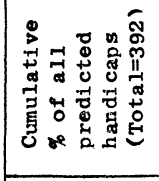 & 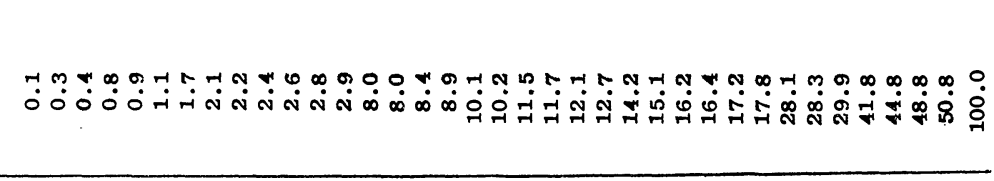 \\
\hline 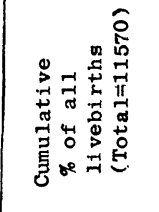 & $\begin{array}{l}0 \\
0 \\
0\end{array}$ \\
\hline 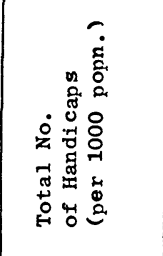 & 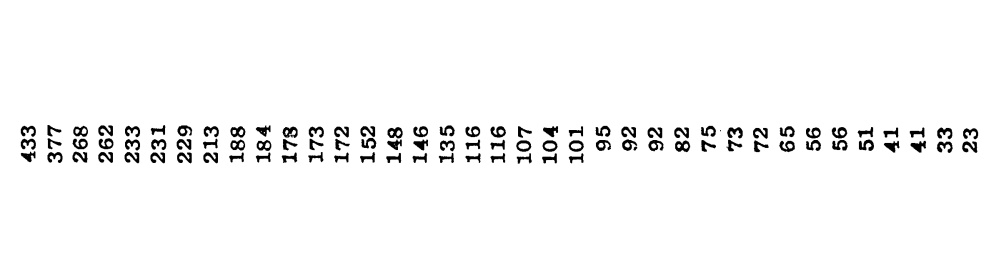 \\
\hline 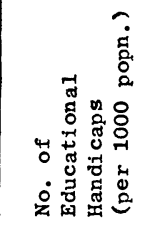 & 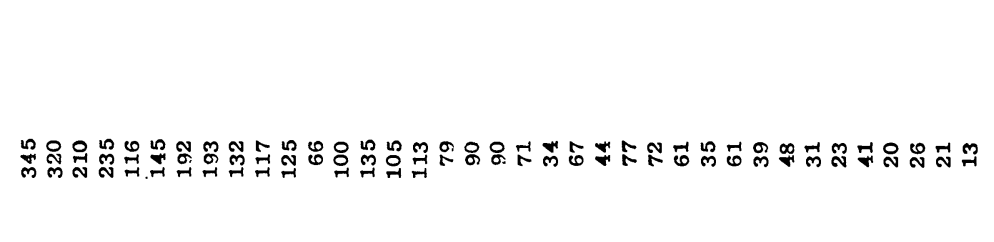 \\
\hline 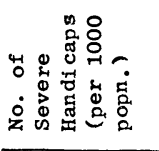 & 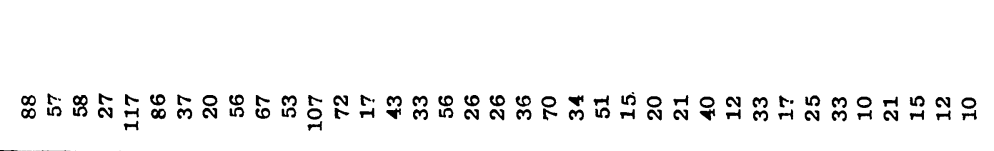 \\
\hline 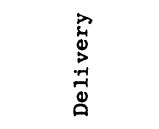 & 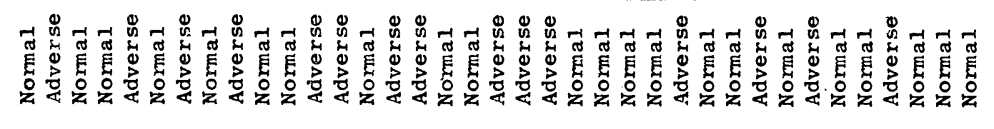 \\
\hline 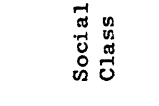 & 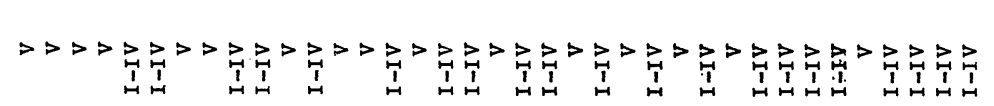 \\
\hline 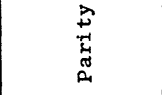 & 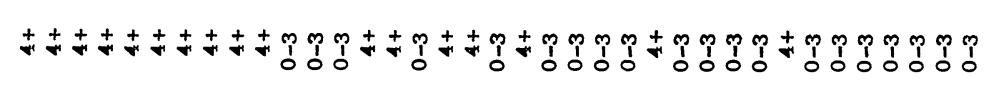 \\
\hline 要尊 & 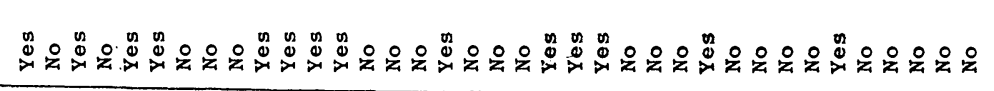 \\
\hline 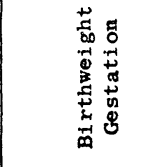 & 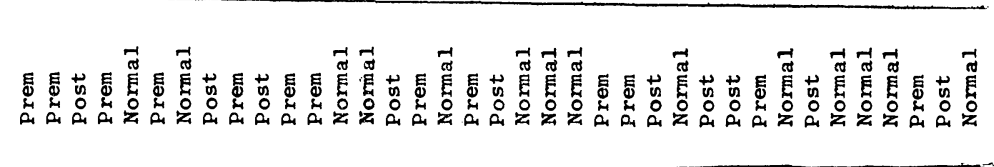 \\
\hline 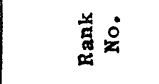 & 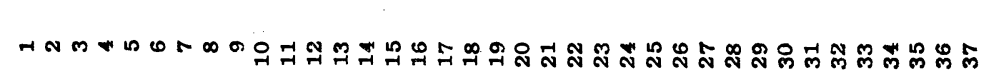 \\
\hline
\end{tabular}




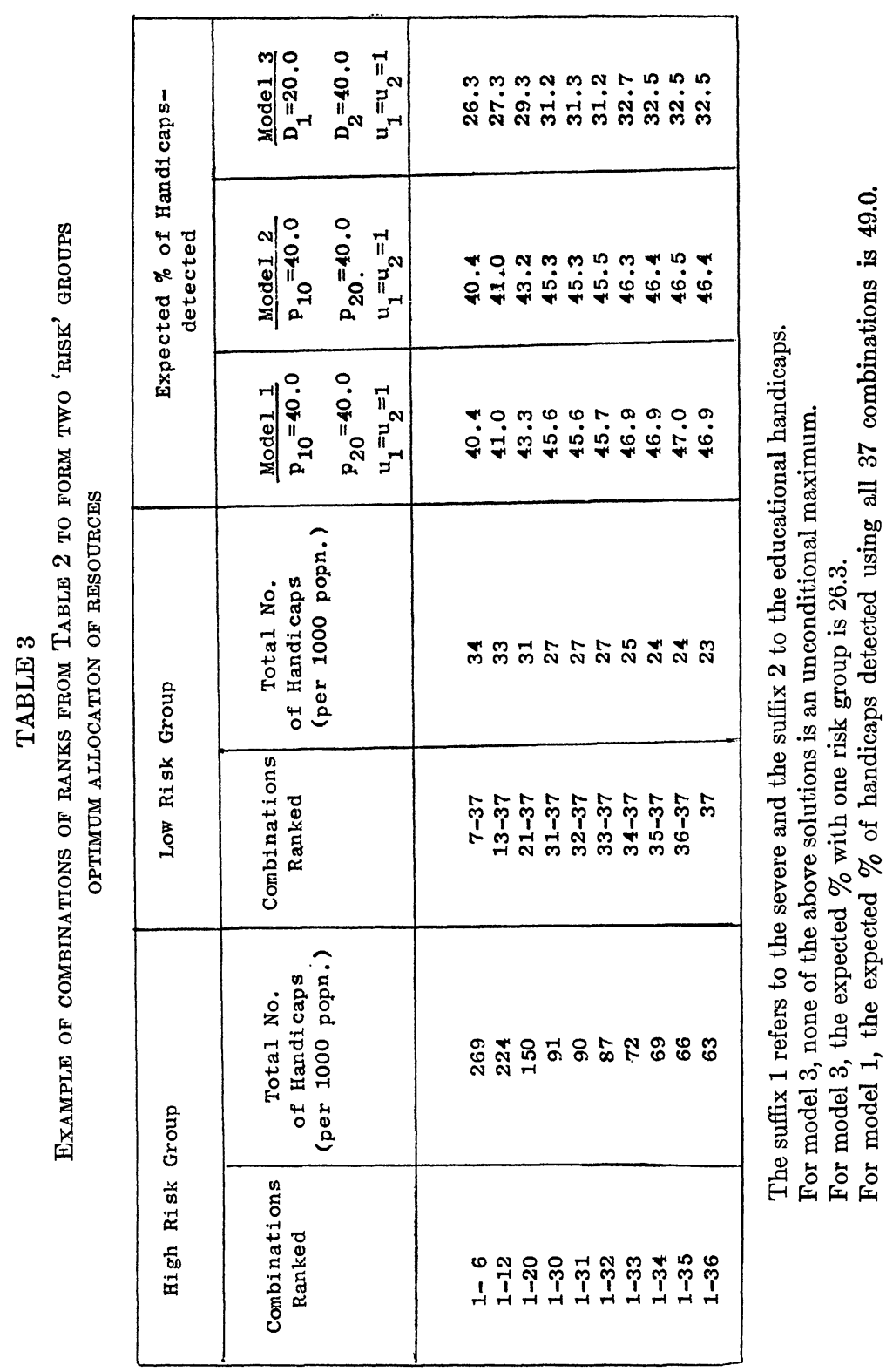


they will be treated together, although in general it should be borne in mind that separate treatment may be better if the restriction of the same set of (perinatal) 'risk' factor combinations for each handicap does not apply. Another drawback of the analysis is the significant result of the test for goodness of fit of the model. Interactions have not been tested, and in some cases are inestimable because of the lack of observations in some of the cells. In particular, it may be expected that there would be interaction among the response variables (handicaps). Since this analysis is used for illustrative purposes only, however, it will be assumed that the model is adequate.

Following the above analysis the population of children can be divided into groups on the basis of the perinatal variables, each group associated with specified risks of developing the two handicaps. Furthermore, since in this example we are attempting to maximise the total number of handicaps, that is using (5), it is useful to rank these groups in order of the total predicted proportion of handicaps in the group. This is done in Table 2 for all 37 combinations of the perinatal variables for which sample observations exist.

The rank order in Table 2 is not the same for the separate handicaps, and the rank order of the sum of the two tends to be more heavily weighted by the educational handicaps, as the average probability of such a handicap is nearly twice that of a severe handicap. Since the ordering in this table forms the basis of the division of the population into a high and a low risk group (discussed below) it might be felt that in practice different weights should be applied to these probabilities, if, say, greater importance were to be attached to detecting a severe rather than an educational handicap. This paper, however, is not the appropriate place to take such issues further.

Table 2 may be used as it stands in order to determine the optimum allocation of resources to all 37 groups. As has already been pointed out, however, interest usually centres on 2 groups only. There are 36 ways of constructing 2 groups from Table 2, such that all the constituent groups in one (the 'high risk' group) have a higher total probability of handicap then all the constituent groups in the other (the 'low risk' group).

For some of these divisions Table 3 shows the expected number of handicaps detected with the optimum allocation of resources under Models 1-3.

For each model arbitrary values of $P_{j 0}$ or $D_{i}$ have been chosen and the expected percentage of detected handicaps under the optimum allocation of resources is calculated for each division. That division which gives the greatest expected percentage of detected handicaps is chosen as the best out of all possible divisions.

It will be seen that the best division for Models 1 and 2 is ranks $1-35$ for the 'high risk' group and for Model 3 is ranks 1-33. It is not in general true that the best division is the same for all values of $P_{j 0}$ or $D_{i}$. Table 4 shows the best division for Model 3 for different combinations of values of $D_{1}$ and $D_{2}$.

It will be seen that where small amounts of total resource are available 
TABLE 4

EXAMPLe OF the 'Best' DIVISIONS INTO tWo GROUPS FOR MODEL 3.

Combinations OF RANKS DERIVED FROM TABLE 2.

\begin{tabular}{|c|c|c|c|}
\hline$D_{1}(\%)$ & $D_{2}(\%)$ & High Risk group & Low Risk group \\
\hline 5.0 & 5.0 & $1-28$ & $29-37$ \\
5.0 & 20.0 & $1-33$ & $34-37$ \\
5.0 & 40.0 & $1-33$ & $34-37$ \\
20.0 & 5.0 & $1-26$ & $27-37$ \\
20.0 & 20.0 & $1-33$ & $34-37$ \\
20.0 & 40.0 & $1-33$ & $34-37$ \\
40.0 & 5.0 & $1-22$ & $23-37$ \\
40.0 & 20.0 & $1-33$ & $34-37$ \\
40.0 & 40.0 & $1-33$ & $34-37$ \\
\hline
\end{tabular}

The suffix 1 refers to the severe and the suffix 2 to the educational handicaps.

(e.g., $D_{1}=5.0, D_{2}=5.0$ ) the division gives a considerably smaller high-risk group than for moderate and large amounts of total resource.

With the choice of $P_{10}$ and $P_{20}$ in Table 3 there does not appear to be much difference between Models 1 and 2. There may, however, be a larger difference for other values of $P_{10}$ and $P_{20}$. Figure 1 compares Models 1 and 2 for a range of values of $P_{20}$ with fixed $P_{10}$.

It will be seen that the advantage of Model 1 is greatest when $P_{10}$ is large. The lower continuous line is the expected percentage of handicaps detected with uniform allocation of resources in an undivided population. It is clear that the greatest relative gain is with small total amounts of resource.

\section{Model 5}

The data for this example are taken from Butler and Alberman [1969] where the probability of a stillbirth or neonatal death was related to variables measured during pregnancy. The analysis found 6 variables to be significant and, as in the previous example, combinations of these may be ranked in order of mortality risk. The first 4 risk combinations are shown in Table 5. It will be assumed that equation (46) is satisfied, and that the probability of preventing mortality is greater if a baby is born in hospital than at home, distinguishing only between these two possibilities. It follows that the babies at lowest risk should be booked for a home delivery. If, for example, there are hospital beds for about $80 \%$ of mothers (the British average), then on this model mothers in the first 3 combinations should have their babies at home. It is seen that, apart from pre-eclampsia, the values 


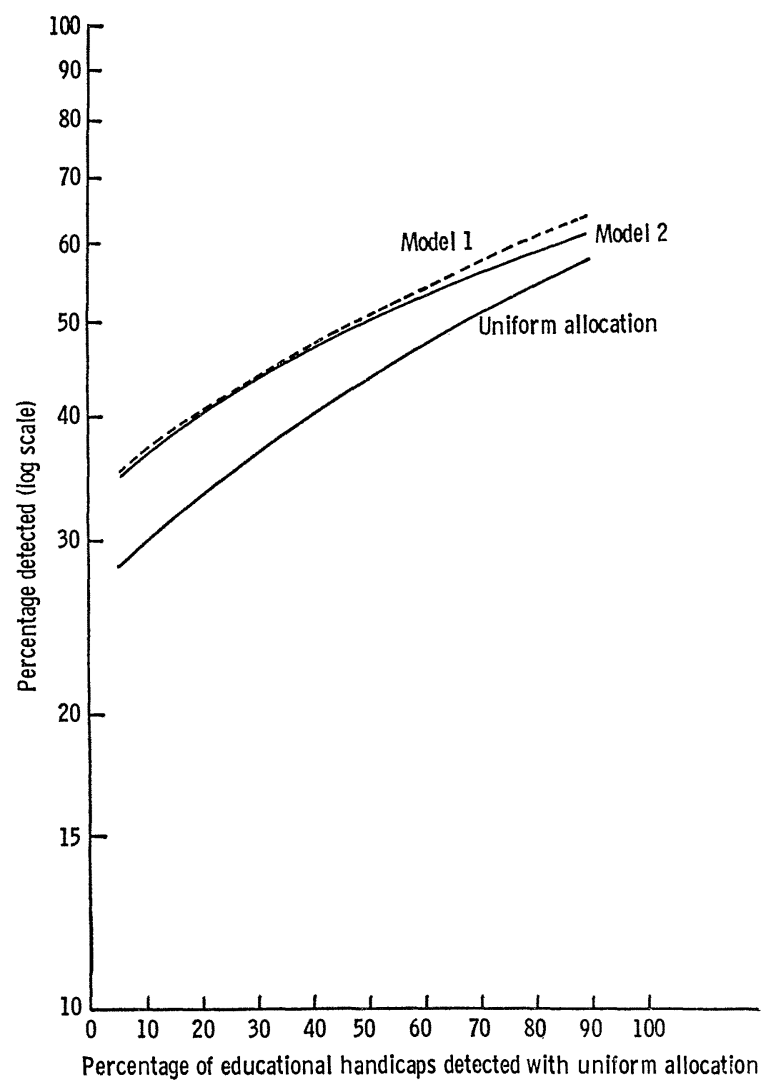

FIGURE 1

EXPECTEd PERCENTAge OF HANDICAPS DETECTED With OPTIMUM ALlOCATION OF REsources for models 1 and 2. Percentage of severe handicaps detected WITH UNIFORM ALLOCATION $=40.0\left(u_{1}=u_{2}=1\right)$

of the other 5 variables are known at the time of booking and an allocation can be made on the basis of these variables. If a mother has been assigned to have a home booking and then develops severe pre-eclampsia, she would immediately be booked for hospital as she would no longer be classified into one of the first 3 combinations.

\section{FURTHER CONSIDERATIONS}

The models presented may be extended in several ways.

Different utility functions could be studied as indicated earlier, in order to see what difference, if any, was made to the optimum allocation of resources. More important, however, is an examination of the form of $g(R)$. It would be useful to study the assumptions underlying the particular form adopted for Models 1-4 and to see what effect different assumptions would have. There seems to be no empirical data relating to this problem, although 
TABLE 5

Maternal Categories in Order of Increasing Risk of Stillbirth or Neonatal Mortality

\begin{tabular}{|c|c|c|c|c|c|c|c|}
\hline $\begin{array}{c}\text { Social } \\
\text { Class }\end{array}$ & Age & Height & Pre-eclampsia & Parity & Smoking & $\begin{array}{l}\text { Cumulative } \\
\% \text { of } \\
\text { Population }\end{array}$ & $\begin{array}{l}\text { Cumulative } \\
\text { Perinatal } \\
\text { Mortality } \\
\text { per } 1000\end{array}$ \\
1 or 2 & $<35$ & $\geqslant 5^{\prime} 2^{\prime \prime}$ & None, mild or moderate & $1-3$ & Non-Smoker & 5.07 & 20.0 \\
3 & $<35$ & $\geqslant 5^{\prime} 2^{\prime \prime}$ & None,mild or moderate & $1-3$ & Non-Smoker & 18.63 & 21.2 \\
1 or 2 & $<35$ & $\geqslant 5^{\prime} 2^{\prime \prime}$ & None, mild or moderate & $1-3$ & Non-Smoker & 19.46 & 22.5 \\
& $\geqslant 35$ & $\geqslant 5^{\prime} 2^{\prime \prime}$ & None,mild or moderate & 0 & Non-Smoker & 23.43 & 22.9 \\
\hline
\end{tabular}

Average perinatal mortality rate $=34.9$ per 1000

N. B. A non-smoker is defined as a mother who smokes less than 1 cigarette a day after the 4 th month of pregnancy.

a current study (Combined Obstetric and Child Health Project, London Borough of Hounslow) is collecting relevant information.

In other applications of decision theory models, especially in medicine, one of the difficulties has been the combination of different types of 'cost' into a single utility. Aitchison [1970] considers such models for allocating treatments to patients. His models deal with the case of continuous final states and two treatments (types of resource) rather than the presence or absence of a state and continuous resources. The principal difference, however, between his approach and the present one seems to lie in the fact that whereas he assumes a given initial state and allocates costs to the different possible treatments, the present approach considers the distribution of this initial state in a population; and the allocation of 'treatments' to the population is made for a fixed total available amount of such treatments. It is this feature of the present approach which avoids the above difficulty in defining the utility function. It may be that the present approach, with suitable modification, could prove useful in some of the situations envisaged by Aitchison.

\section{ACKNOWLEDGMENTS}

I would like to thank the National Birthday Trust Fund and the Directors and Steering Committee of the National Child Development Study for permission to publish results of the study; also Dr. E. D. Alberman, Mr. M. J. R. Healy, and Professor J. M. Tanner for their help and encouragement, and Professor R. Darrell Bock for the use of his multinomial response computer program. I am grateful to a referee for drawing my attention to the paper by Brunk [1958]. This work was supported by a grant from the Nuffield Foundation to the Department of Growth and Development at the Institute of Child Health. 


\section{LA REPARTITION DES MOYENNES DANS UN SCREENING DE POPULATION: UN MODELE DECISIONNEL}

\section{RESUME}

Dans le screening médical d'une population pour des maladies latentes, il est souvent possible de définir des sous-populations différant dans les risques des maladies. Dans le but de rendre la plus efficace possible l'utilisation des ressources disponibles pour les programmes de screening, l'attribution de ces ressources devrait prendre en compte les différents risques. Quelques modèles relevant de la théorie de la décision sont proposés pour déterminer les répartitions optimales. Ces modèles sont appliqués à des données sur les handicaps chez des enfants jeunes et à la mortalité périnatale.

\section{REFERENCES}

Aitchison, J. [1970]. Statistical problems of treatment allocation. J. R. Statist. Soc. A. 198, 206-39.

Alberman, E. D. and Goldstein, H. [1970]. The 'at risk' register: a statistical evaluation. Brit. J. Prev. Soc. Med. 24, 129-35.

Aubry, R. H. and Nesbitt, R. E. L. [1969]. High-risk obstetrics. Amer. J. Obst. Gynec. 105, $241-7$.

Bock, R. D. [1969]. Estimating multinomial response relations. In: Contributions to Statistics and Probability: Essays in Memory of S. N. Roy. Bose, R. C. (Ed.), University of North Carolina Press, Chapel Hill, N. C.

Brunk, H. D. [1958]. On the estimation of parameters restricted by inequalities. Ann. Math. Statist. 29, 437-54.

Butler, N. R. and Alberman, E. D. (Eds.). [1969]. Perinatal Problems. Livingstone, Edinburgh.

Davie, R., Butler, N. R., and Goldstein, H. [1972]. From Birth to Seven. Longmans, London.

Geary, R. C. and McCarthy, M. D. [1964]. Elements of Linear Programming. Griffin, London.

$\rightarrow$ Goodman, L. A. [1970]. The multivariate analysis of qualitative data: interactions among multiple classifications. J. Amer. Statist. Ass. 65, 226-56.

Lindon, R. L. [1961]. Risk register. Cerebral Palsy Bulletin 3, 481-7.

\section{APPENDIX}

In Models 1-4, the unconditional maximum solution may give values of some of the $R_{j i}$ which are negative. The relative maximum of the expected utility is the maximum subject to all $R_{j i} \geq 0$.

Consider the $n$-dimensional Euclidean space defined by the $R_{j i}$. If equation (3) is used, $n=k$ and each resource $j$ may be considered separately. If equation (4) is used $n=k s$. The conditions (3) and (4) each define a hyperplane of dimension $n-1$ which contains all solutions.

Since $C$ is a linear combination of convex functions of the form $1-e^{-R}$, $C$ is also convex. It is shown by Brunk [1958] that for such a function, if it has an absolute maximum outside the feasible region determined here by equation (10), then the relative maximum lies on the boundary of this region, that is, when one or more $R_{j i}$ are equal to zero.

\section{Received December 1970, Revised September 1971}

Key Words: Screening; Handicaps in childhood; Perinatal mortality; Risk register; Resource allocation; Decision theory; Multivariate logit analysis. 